\title{
An optical angle sensor based on chromatic dispersion with a mode-locked laser source
}

\author{
Yuki SHIMIZU*, Shuhei MADOKORO*, Hiraku MATSUKUMA* and Wei GAO* \\ *Department of Finemechanics, Tohoku University \\ 6-6-01, AramakiAza Aoba, Aoba-ku, Sendai, Miyagi 980-8579, Japan \\ E-mail: yuki.shimizu@nano.mech.tohoku.ac.jp
}

Received: 29 March 2018; Revised: 3 July 2018; Accepted: 25 July 2018

\begin{abstract}
This paper proposes a new optical angle sensor, in which a mode-locked laser is employed as the light source, and chromatic dispersion of a collimator objective is utilized to detect the angular displacement of a target of interest. In the proposed method, each of the optical modes in the femtosecond laser beam reflected from the reflector mounted on a target of interest is separated from the others by chromatic dispersion of a collimator objective to generate a group of focused laser beams that can be utilized as the scale for measurement of an angular displacement of the target. By detecting the change in peak frequency in optical spectra obtained by the photodetector, a small angular displacement can be measured. In this paper, as the first step of research, a prototype optical setup is developed, and some basic experiments are carried out to demonstrate the feasibility of the proposed method for measurement of angular displacement.
\end{abstract}

Keywords : Optical frequency comb, Angular displacement, Angle measurement, Chromatic dispersion, Laser autocollimation

\section{Introduction}

Precision positioning system plays one of the important roles in precision machine tools for fabrication of fine optical elements, semiconductor devices and so on. To achieve nanometric positioning accuracy, which is required not only for the stage system employed in the state-of-the-art scientific instruments but also for commercial machine tools, most of the precision positioning systems are controlled in a closed loop with displacement sensors such as linear encoders or laser interferometers (Gao et al., 2015). With the employment of displacement sensors, translational error motion of a stage table can be corrected. Meanwhile, angular error motion of a stage table, which could cause positioning error referred to as the Abbe error (Bryan, 1979), has not been measured in most of the cases. Since it is not so easy to satisfy the Abbe principle in most of the machine tools, it is essential to measure the angular error motion of a stage table for achieving further higher positioning accuracy (Gao et al., 2015).

For measurement of angular error motion of a stage table, optical angle sensors based on the laser autocollimation (Ennos et al., 1982), which can carry out measurement of small angular displacement, are often employed. In the laser autocollimation, the angular displacement of a target of interest can be obtained by detecting the translational displacement of a reflected laser beam on the focal plane of a collimator objective. Figure 1 shows a generalized optical setup for an optical angle sensor based on the laser autocollimation. The setup is mainly composed of a light source, a reflector to be mounted on a target of interest, a collimator objective and a photodetector. In the setup, a collimated laser beam is made incident to the reflector, and the reflected laser beam is made to focus on the photodetector placed on the back focal plane of the collimator objective. The relationship between the angular displacement $\Delta \theta$ of the reflector and the translational displacement $\Delta d$ of the focused laser beam on the photodetector can be expressed as follows (Ennos et al., 1982): 


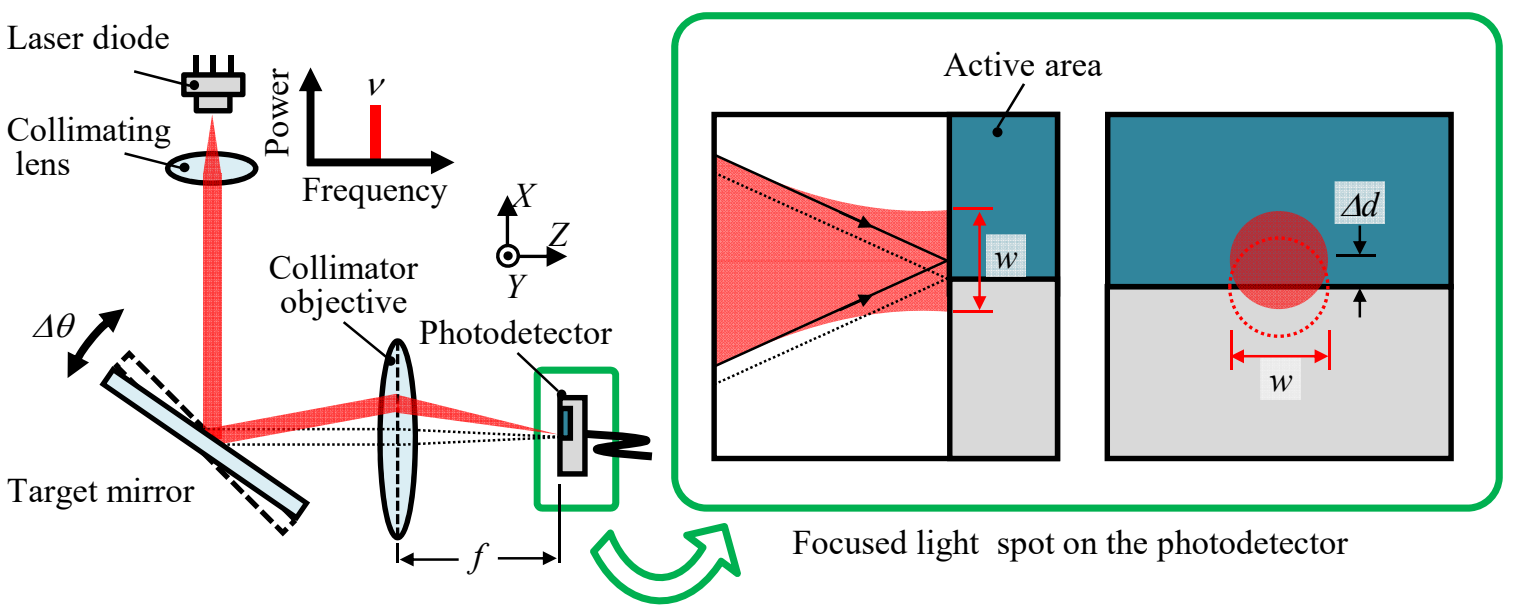

Fig. 1 A setup for an optical angle sensor based on the laser autocollimation employing a single-cell photodiode.

$$
\Delta \theta=\frac{1}{2} \tan ^{-1} \frac{\Delta d}{f}
$$

Where $f$ is a focal length of the collimator objective. With the employment of a charge coupled device (CCD) as the photodetector of an optical angle sensor based on the laser autocollimation, a wide measurement range of over 1000 arc-seconds can be achieved (Ishikawa et al., 2014), while its measurement sensitivity becomes in proportion to $f$. On the other hand, in the case where a photodiode is used as the photodetector, the measurement sensitivity becomes independent from $f$, and an optical setup based on the laser autocollimation can be designed in a compact size (Saito et al., 2007). According to the previous study by the author's group, the sensor sensitivity $S$, which represents the degree of the change in photodiode output with respect to the change in angular displacement of a target of interest, becomes independent from $f$ and can be given by the following equation (Saito et al., 2007; Gao, 2010):

$$
S \propto \frac{D v}{c}
$$

where $D$ is the beam diameter made incident to the collimator objective, $c$ is the light speed in air, and $v$ is the light frequency of the laser beam. The above equation shows that the sensitivity $S$ can be improved by increasing $D$. Based on this concept, a high resolution optical angle sensor having a resolution of better than 0.001 arc-second has been developed (Shimizu et al., 2016). Meanwhile, as can be seen in Eq. (2), the fluctuation of light frequency $\Delta v$, which is often observed in the laser beam emitted from a laser diode due to the change in temperature up to $20 \mathrm{GHz} /{ }^{\circ} \mathrm{C}$ (Saito et al., 1982), could result in the instability of sensor sensitivity $S$ as shown in Fig. 2. Another drawback of the conventional optical angle sensors based on the laser autocollimation utilizing photodiodes is its narrow measurement

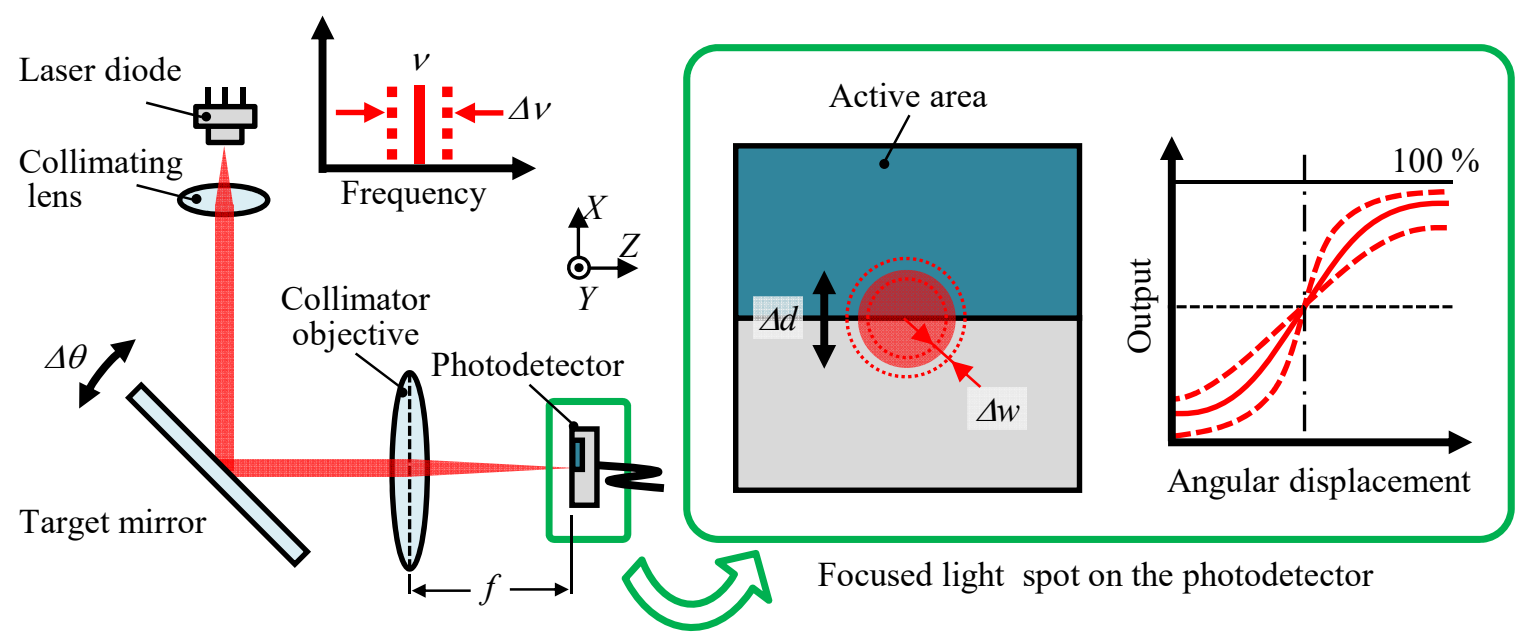

Fig. 2 Instability of the optical angle sensor sensitivity induced by the fluctuation of laser frequency. 
range, which is determined by a diameter of the focused laser spot on the active cell in a photodiode, limited to the order of only 100 arc-seconds (Chen et al., 2017).

Meanwhile, recently, a mode-locked laser often referred to as the optical frequency comb (Holzwarth et al., 2000) is attracting much attentions in many scientific and industrial fields (Tan et al., 2005; Moreaux et al., 2000; Kim, 2009; Chong et al., 2008; Minoshima et al., 2000) due to its precise and highly stable optical frequencies. To overcome the above mentioned drawbacks of the conventional optical angle sensor based on the laser autocollimation employing photodiodes and a laser diode, an attempt has been made to employ a mode-locked femtosecond laser beam as the light source for the optical angle sensor (Tamada et al., 2016). In addition, a femtosecond laser autocollimator, which employs a femtosecond laser source as the light source for the laser autocollimator, has also been developed (Chen et al., 2017). With the employment of a grating reflector, a measurement range has successfully been extended to larger than 11,000 arc-seconds while employing a photodiode as the photodetector. Furthermore, a much wider measurement range of larger than 21,600 arc-second with an increased visibility of output signal of the femtosecond laser autocollimator has also been achieved by the optical frequency domain angle measurement method (Chen et al., 2017). Meanwhile, one of the drawbacks of the mode-locked laser autocollimator is that the employment of a high-precision reflective-type diffraction grating, which is to be mounted on a target of interest, is essential to obtain a group of the first-order diffracted beams required for the detection of angular displacement.

In responding to the background described above, in this paper, a new angle sensor with a mode-locked laser source is proposed to achieve measurement of an angular displacement without employing a grating reflector. The proposed angle sensor measures the angular displacement of a target of interest by combining the mode-locked femtosecond laser and the chromatic dispersion of a collimator objective employed in the setup of the optical angle sensor to generate a group of focused laser beams that can be utilized as the scale for measurement of an angular displacement. In this paper, as the first step of research, attentions are paid to confirm the possibility of realizing the proposed method for measurement of angular displacements. After a description of the proposed principle of the optical angle sensor, results of the experiments with a developed prototype optical angle sensor are presented.

\section{Principle of the proposed method for measurement of an angular displacement}

In the proposed method, a mode-locked laser, which is often referred to as the optical frequency comb, is combined with the chromatic dispersion of a collimator objective. It is well known that the frequency $v_{i}$ of the $i$ th optical mode can be expressed by the following equation (Jones et al., 2000):

$$
v_{i}=i v_{\text {rep }}+v_{\mathrm{CEO}}
$$

where $v_{\text {rep }}$ and $v_{\text {CEO }}$ are the pulse repetition rate and the carrier envelope offset frequency, respectively, the both of which can be stabilized by using a frequency standard such as the rubidium $(\mathrm{Rb})$ frequency standard. A mode-locked laser can therefore be utilized as the light source having a broadband spectrum with highly accurate optical mode frequencies. Now we consider the case where a mode-locked laser beam is made incident to a collimator objective having chromatic aberration as shown in Fig. 3. For the sake of simplicity, the laser axis is set to be parallel with the optical axis of the collimator objective. Each of the optical modes in the optical frequency comb made incident to a lens is refracted with a different angle of refraction due to the influence of chromatic dispersion, and propagates in a direction different from the others after passing through the collimator objective. The relationship between the angle of incidence $\theta_{\text {inc }}$ and that of refraction $\theta_{\text {ref }}$ of the $i$ th optical mode with the wavelength $\lambda_{i}$, which corresponds to the optical frequency $v_{i}$, can be expressed by the following equation:

$$
n_{0} \sin \theta_{\text {inc }}=n\left(\lambda_{i}\right) \sin \theta_{\text {ref }}
$$

where $n_{0}$ is the refractive index of air, and $n\left(\lambda_{i}\right)$ is the refractive index of lens material with respect to the light wavelength $\lambda_{i}$ that can be expressed as follows:

$$
n\left(\lambda_{i}\right)^{2}=1+\frac{B_{1} \lambda_{i}^{2}}{\lambda_{i}^{2}-C_{1}}+\frac{B_{2} \lambda_{i}^{2}}{\lambda_{i}^{2}-C_{2}}+\frac{B_{3} \lambda_{i}^{2}}{\lambda_{i}^{2}-C_{3}}
$$




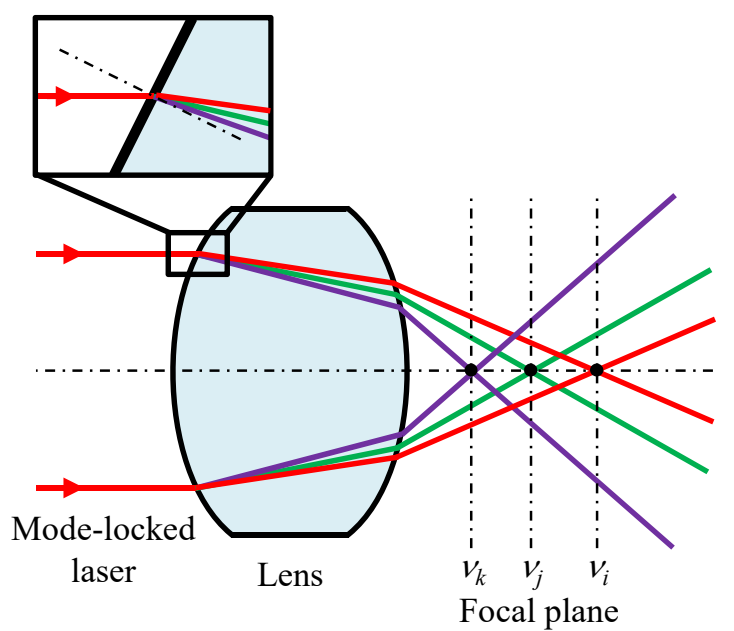

Fig. 3 Effect of the chromatic dispersion on the focal length of the collimator objective.

where $B_{m}$ and $C_{n}(m, n=1,2,3)$ are the Sellmeier coefficients determined by the material of collimator objective (Hecht, 2017). According to the thin-lens equation, in the case of plano-convex lens, the focal length $f_{i}$ of the collimator objective with respect to $\lambda_{i}$ can be expressed as follows (Hecht, 2017):

$$
\frac{1}{f_{i}}=\left(n\left(\lambda_{i}\right)-1\right)\left(\frac{1}{R_{1}}-\frac{1}{R_{2}}\right)=\left(\frac{B_{1} \lambda_{i}^{2}}{\lambda_{i}^{2}-C_{1}}+\frac{B_{2} \lambda_{i}^{2}}{\lambda_{i}^{2}-C_{2}}+\frac{B_{3} \lambda_{i}^{2}}{\lambda_{i}^{2}-C_{3}}\right)\left(\frac{1}{R_{1}}-\frac{1}{R_{2}}\right)
$$

where $R_{1}$ and $R_{2}$ represent the surface radii of the collimator objective. It should be noted that the influences of monochromatic aberrations such as the spherical aberration are neglected for the sake of simplicity. As can be seen in the above equation, each of the light rays with a different frequency (, and hence a different wavelength) will have a different focal length as shown in Fig. 3. In the proposed method, this phenomenon is utilized to detect the small angular displacement of a target of interest.

Figure 4 shows a schematic of the optical setup for the optical angle sensor based on the proposed method. A mode-locked laser source is employed as the light source, while an optical fiber with an optical spectrum analyzer is employed as the photodetector. Now we give a known initial inclination angle $\theta$ to a reflector, while placing the end of

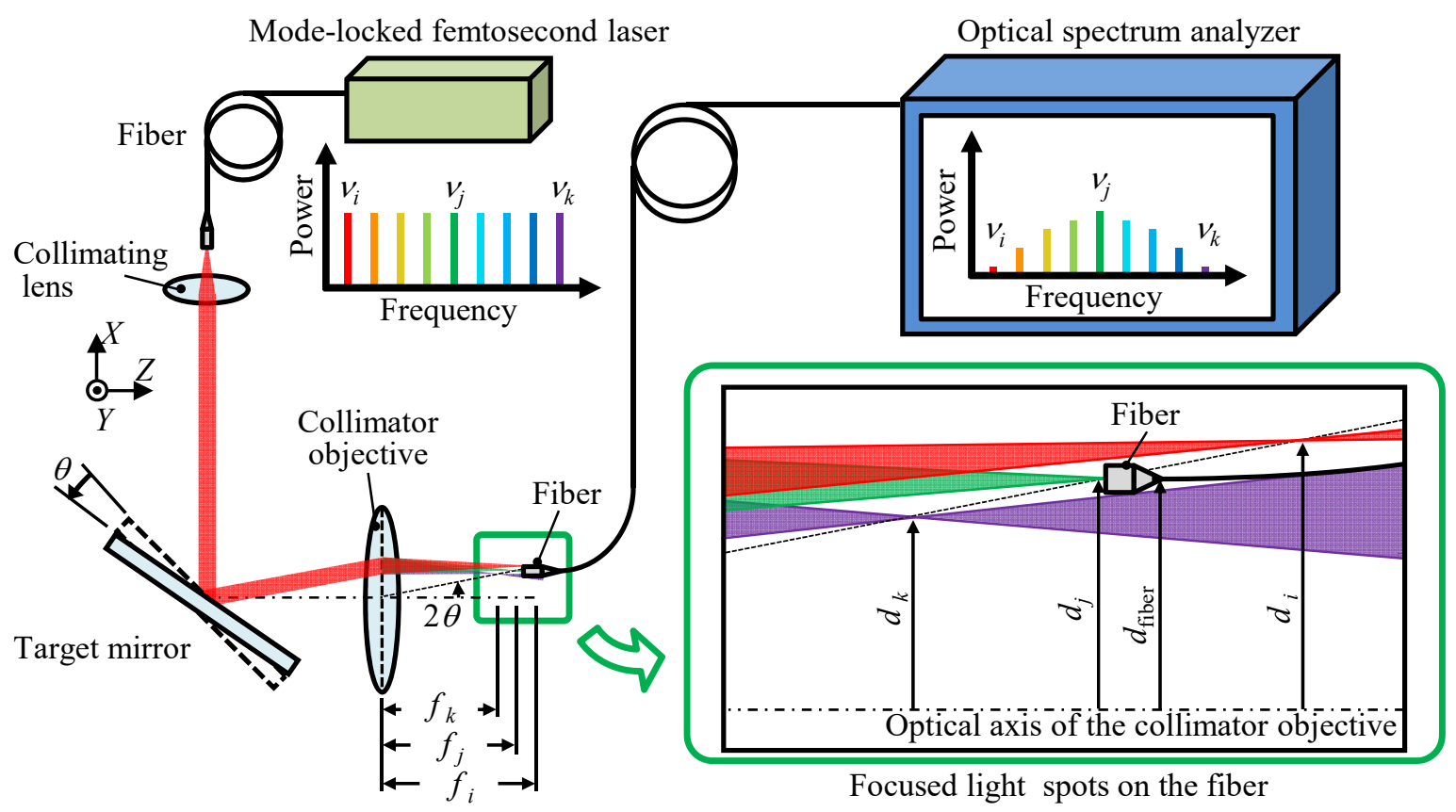

Fig. 4 A schematic of the optical setup for the proposed method for measurement of angular displacement. 
the optical fiber with a distance $d_{\text {fiber }}$ with respect to the optical axis of the collimator objective in the plane of the figure. It should be noted that $\theta$ is determined in such a way that the axis of reflected femtosecond laser beam becomes parallel with the optical axis of the collimator objective when $\theta=0$. The collimated femtosecond laser beam from the light source is made incident to a target mirror, and the reflected femtosecond laser beam is made incident to a collimator objective. It should be noted that the collimator objective employed in the setup is not well corrected with respect to the chromatic aberration. In the setup, the optical axis of the collimator objective has an angular offset $2 \theta$ with respect to the reflected femtosecond laser beam. Due to the influence of chromatic dispersion of the collimator objective, each optical mode in the mode-locked femtosecond laser will be focused at the position corresponding to its optical frequency (hence, its light wavelength). Based on the principle of laser autocollimation, a position $d_{i}$ of the focused $i$ th optical mode with respect to the optical axis of the collimator objective can be expressed by the following equation:

$$
d_{i}=f_{i} \tan 2 \theta
$$

Now we consider the case where each of the optical modes in the reflected femtosecond laser has the same light intensity over its spectrum, and the light intensity of $j$ th optical mode is the largest in the spectrum captured by the optical spectrum analyzer through the optical fiber when $\theta$ is set to be $\theta_{0}(\neq 0)$. In this case, the following relationship can be found:

$$
d_{\text {fiber }}=d_{j}=f_{j} \tan 2 \theta_{0}
$$

Giving a small angular displacement $\Delta \theta$ will generate displacements of all the focused optical modes, and now we consider the light intensity of $k$ th optical mode becomes the largest in the spectrum captured by the optical spectrum analyzer. In the same manner as described above, the following equation can be found:

$$
d_{\text {fiber }}=d_{k}=f_{k} \tan 2\left(\theta_{0}+\Delta \theta\right)
$$

From Eqs. (8) and (9), the angular displacement $\Delta \theta$ can be obtained by the following equation:

$$
\Delta \theta=\frac{1}{2} \tan ^{-1}\left(\frac{f_{j}}{f_{k}} \tan 2 \theta_{0}\right)-\theta_{0}
$$

On the assumption that $\theta_{0}$ is known as the design parameter of the optical setup, since $f_{j}$ and $f_{k}$ can be calculated based on Eq. (6) by using the detected peak frequencies $v_{j}$ and $v_{k}$, the angular displacement $\Delta \theta$ can be obtained from Eq. (10). It should be noted that, as the first step of research, this paper has focused on the proposal and verification of the measurement method, and $\theta_{0}$ has been treated as a known parameter. As can be seen in Eq. (10), the determination of $\theta_{0}$ is important for accurate measurement of the angular displacement $\Delta \theta$, and will be carried out in future work as the next step of research.

\section{Experiments}

In order to verify the feasibility of proposed method, an optical setup was developed as shown in Fig. 5. In the setup, a commercial mode-locked femtosecond laser source (C-Fiber, MenloSystems GmbH) was employed as the light source. The femtosecond laser beam emitted from the laser source had an optical spectrom ranging from $185 \mathrm{THz}$ to $200 \mathrm{THz}$, and its pulse repetition rate was $100 \mathrm{MHz}$. The femtosecond laser beam emitted from the light source was at first collimated by a collimating lens, and was then made incident to a mirror reflector mounted on a piezoelectric (PZT) tilt stage system (PSH2z, Piezosystemjena Inc.). A reflected femtosecond laser beam was then focused on an edge of a single-mode optical fiber, whose mode field diameter was $10.4 \mu \mathrm{m}$, by using a collimator objective having a focal length of $15.37 \mathrm{~mm}$ (for $\lambda=656 \mathrm{~nm}$ ). According to the light diffraction theory, beam diameters of the optical modes in the femtosecond laser at the focal plane of the collimator objective were ranging from $17.0 \mu \mathrm{m}$ to $18.4 \mu \mathrm{m}$. The laser beam coupled into the fiber was then analyzed by an optical spectrum analyzer (AQ6370C, YOKOGAWA Co.) so as to acquire the optical spectrum. In the experiment, a frequency resolution bandwidth of the optical spectrum analyzer was set to be $200 \mathrm{GHz}$. The target mirror and the PZT tilt stage were aligned in such a way that the reflected laser beam was made incident to the collimator objective with an inclination angle $\theta_{0}(\neq 0)$ with respect to the optical 


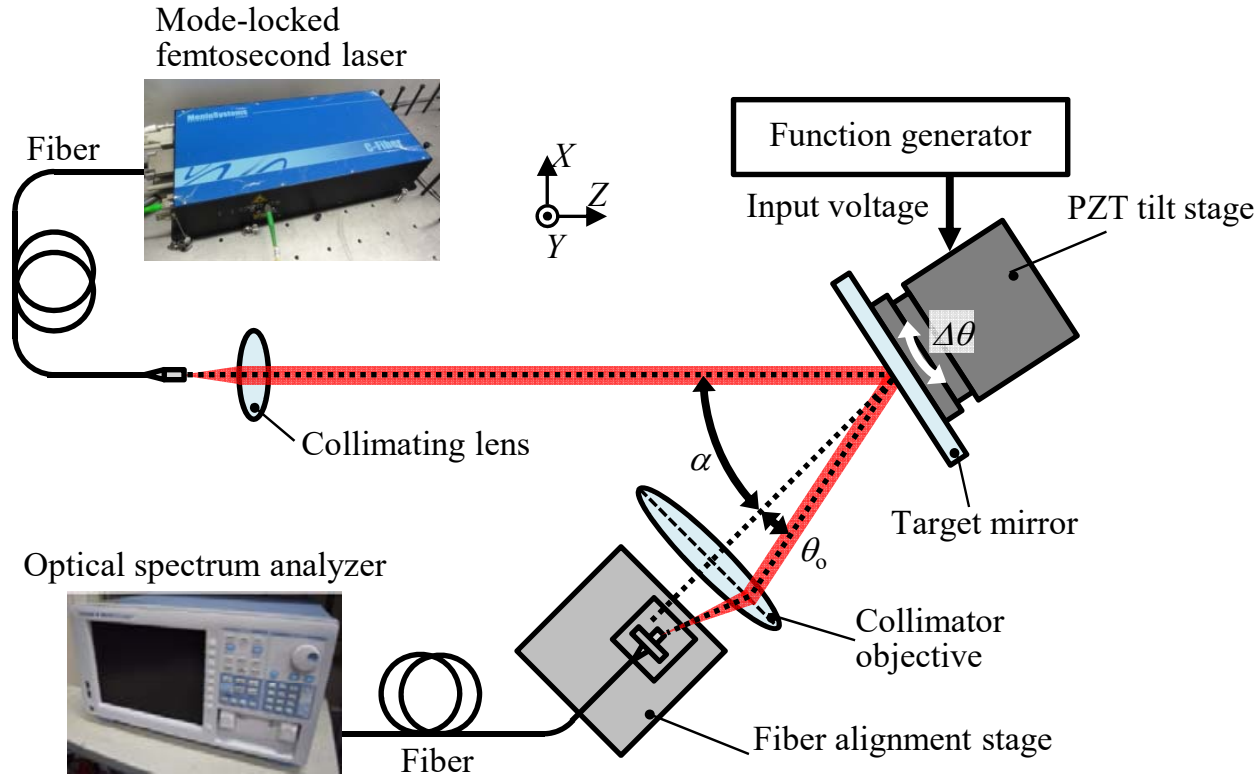

Fig. 5 A schematic of the experimental setup.

Table 1 Design parameters for the optical setup.

\begin{tabular}{cc}
\hline Component & Value \\
\hline Beam diameter $D$ & $3.3 \mathrm{~mm}$ \\
Center wavelength & $1560 \mathrm{~nm}$ \\
Pulse repetition frequency $v_{\text {rep }}$ & $100 \mathrm{MHz}$ \\
Spectral width & $>25 \mathrm{~nm}$ \\
Mode field diameter & $10.4 \pm 0.5 \mathrm{~mm} @ 1550 \mathrm{~nm}$ \\
Focal length of the collimator objective & $15.37 \mathrm{~mm}$ (for $\lambda=656 \mathrm{~nm})$ \\
Resolution of detection frequency & $200 \mathrm{GHz}$ \\
\hline
\end{tabular}

axis of the collimator objective. The design parameters for the developed setup are summarized in Table 1. It should be noted that an objective lens designed for microscopes was employed in the setup as the collimator objective in order to minimize the influences of monochromatic aberrations such as spherical aberration, coma aberration or astigmatism, which cause the deviation of the light spot position (Claus et al., 2011), since the detection of the center position of light spot is important for the proposed method.

Experiments were carried out by using the developed optical setup. Since the chromatic aberration of the collimator objective was utilized in the proposed angle measurement method, at first, an experiment was carried out to confirm that the collimator objective employed in the setup had an ability of generating chromatic dispersion along its

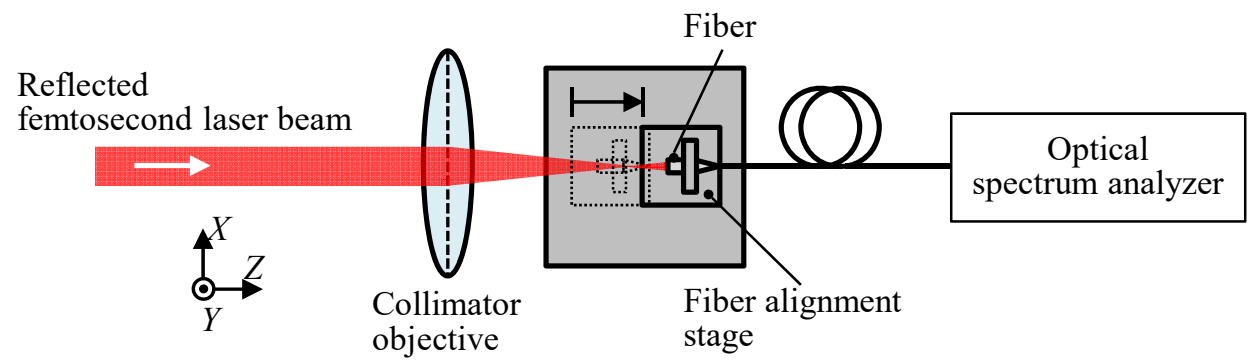

Fig. 6 An optical setup for evaluation of the chromatic dispersion of the collimator objective. 


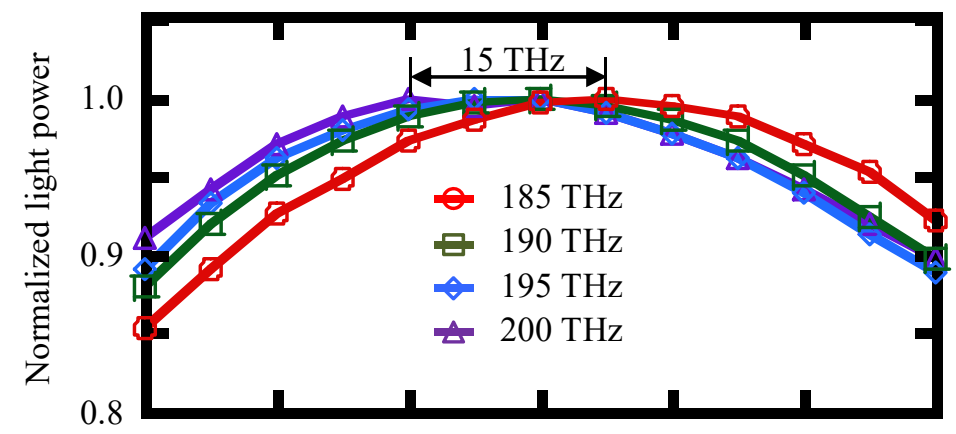

Z-position of the fiber edge $10 \mu \mathrm{m} / \mathrm{div}$.

Fig. 7 Relationship between the Z-directional displacement of the fiber edge and the power of each optical frequency component in the captured laser beam.

optical axis. Figure 6 shows a schematic of the experimental setup. In the constructed optical system, the angular position of the mirror and the position of fiber edge were aligned so that the axis of the reflected femtosecond laser beam, optical axis of the the collimator objective and the fiber axis would coincide with each other. In the experiment, the edge of fiber was moved along the $Z$-direction in a step of $5 \mu \mathrm{m}$ by the fiber alignment stage, and the optical spectrum of the laser beam coupled into the fiber was captured by the optical spectrum analyzer at each $Z$-position of the fiber edge. Figure 7 shows the relationship between the Z-displacement of the fiber edge and the detected light intensities of four different optical frequency components. In the figure, light powers normalized by the maximum light powers obtained at each frequency component are plotted in order to cancel the influences of non-uniform spectrum in the light source. As can be seen in the figure, a chromatic dispersion of approximately $15 \mu \mathrm{m}$ was found between the frequency components of $185 \mathrm{THz}$ and $200 \mathrm{THz}$. From this result, the collimator objective employed in the setup was confirmed to give enough chromatic dispersion to the femtosecond laser beam employed in this paper.

After the verification of chromatic dispersion by the employed collimator objective, experiments were extended to verify the feasibility of proposed angle measurement method. In the experiment, a small angular displacement $\Delta \theta$ in a step of 3.3 arc-seconds was applied to the target mirror by using the PZT tilt stage, and variation of the optical spectrum from $185 \mathrm{THz}$ to $200 \mathrm{THz}$ was measured by the optical spectrum analyzer at each angular position of the mirror reflector. In the experiments, the frequency resolution bandwidth of the optical spectrum analyzer was set to be $200 \mathrm{GHz}$. According to the proposed principle, the light spots generated by the optical frequency comb are dispersed corresponding to the optical frequency by the chromatic aberration of the collimator objective. Therefore, the larger light spot interval was suitable for verification of the proposed principle. It is obvious from Eq. (10) that the light spot interval on the detector becomes larger by increasing initial incident angle $\theta_{0}$. Therefore, in this experiment, the amount of $\theta_{0}$ was set to be as large as possible in order to acquire large spot interval $h_{i}$. It should be noted that the angle $\alpha$ between the axis of the incident femtosecond laser beam and the optical axis of collimator objective and the initial incident angle $\theta_{0}$ were treated as known design parameters. Meanwhile, $\alpha$ will not affect the measurement as long as the measurand is the relative angular displacement of a target of interest. In the following experiments, $\theta_{0}$ was treated to be approximately 5.0 degrees.

Figure 8 shows the relationship between the angular displacement $\Delta \theta$ of the mirror reflector and the changes in measured light power of the four optical frequency components. In the same manner as the result shown in Fig. 7, the changes in light power of are normalized in Fig. 8. As can be seen in the figure, the angular positions giving maximum light power were found to be different for each of the optical frequency components. This was caused by the chromatic dispersion of the collimator objective. These experimental result demonstrated the feasibility of proposed angle measurement method combining the optical frequency comb with the chromatic dispersion of the collimator objective.

Further detailed analyses on the data obtained in the above mentioned experiment were also carried out to estimate a resolution of the angle measurement by the proposed method. Figure 9 shows the angular displacement $\Delta \theta$ giving maximum light power in each frequency component. In the figure, the angular displacement giving maximum light power was determined by fitting a quadratic function to the change in normalized light power of each of the frequency components. As can be seen in Fig. 9, the angle detection sensitivity in the experiment setup was evaluated to be 0.88 $\mathrm{THz} / \mathrm{arc}$-second from the slope of the linear approximation; namely, when an angular displacement of 1 arc-second is given to the mirror reflector, the change in peak optical frequency of $0.88 \mathrm{THz}$ can be observed in the optical spectrum 


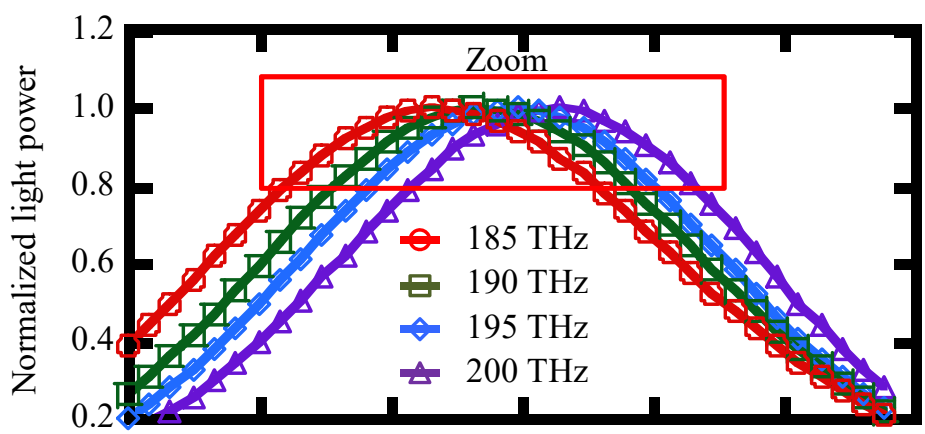

Angular displacement applied to the mirror reflector $20 \mathrm{arc}-\mathrm{second} / \mathrm{div}$.

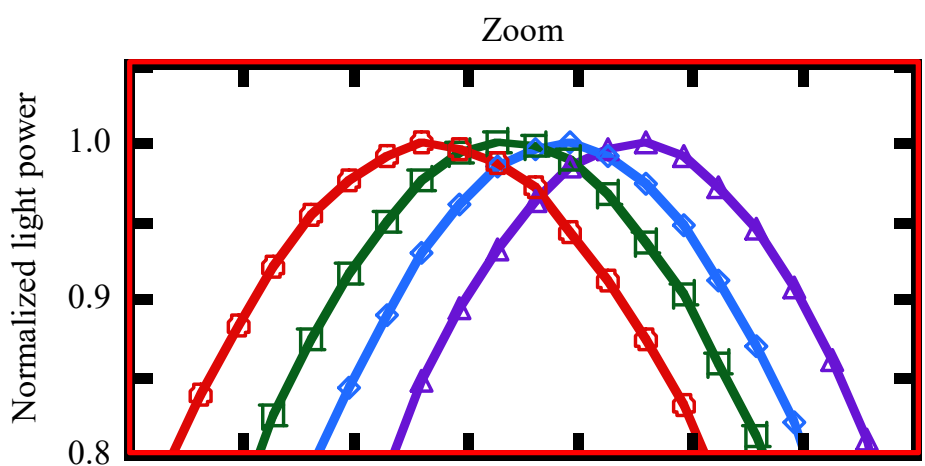

Angular displacement applied to the mirror reflector $10 \mathrm{arc}-\mathrm{second} / \mathrm{div}$.

Fig. 8 The change in power of each optical mode captured by the optical spectrum analyzer due to the angular displacement given to the mirror reflector.

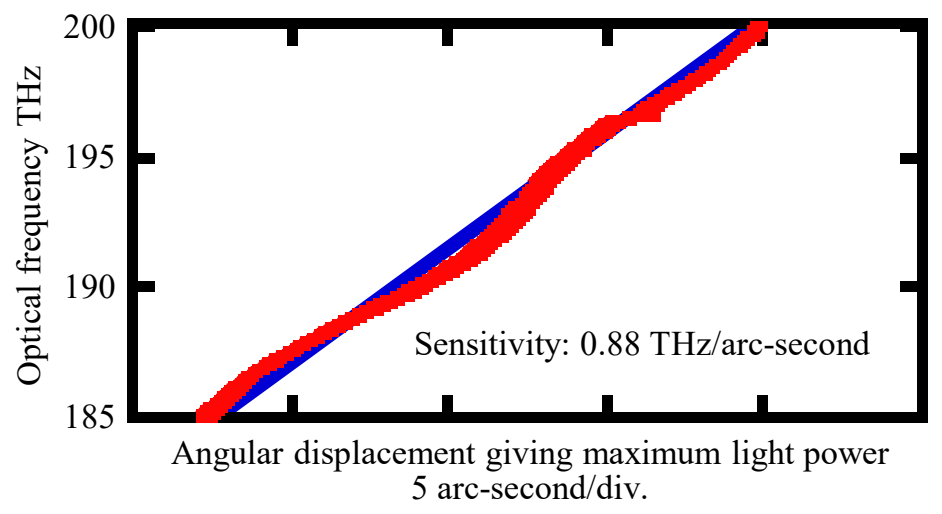

Fig. 9 Estimation of the measurement sensitivity of angular displacement by the proposed method.

captured by the optical spectrum analyzer. From the obtained sensitivity, under the condition of a frequency resolution bandwidth of the optical spectrum analyzer of $200 \mathrm{GHz}$, the measurement resolution of the developed angle sensor was estimated to be approximately 0.23 arc-second. Although the resolution obtained by the developed prototype optical setup is lower than that expected by the conventional laser autocollimators, a further optimization of the optical setup including the employment of a collimator objective having further larger effect of chromatic dispersion is expected to improve the resolution.

It should be noted that, in this paper, the initial incident angle $\theta_{0}$ has been treated as a known parameter in the optical setup. In addition, the experiments have been done in a limited angular displacement due to the limitation on the developed experimental setup employing the PZT tilt stage having a small stroke for giving an angular displacement to the mirror reflector. For the quantitative evaluation of the sensor characteristics, a modification of the experimental 
setup by using a precision rotary table having a larger stroke is required, and will be carried out in future work.

\section{Conclusions}

A new optical angle sensor, in which a mode-locked laser is employed as the light source, and chromatic dispersion of a collimator objective is utilized to detect the angular displacement of a target of interest, has been proposed. The proposed method has been designed in such a way that each optical mode in a reflected femtosecond laser beam is separated by chromatic dispersion of a collimator objective to generate a group of focused laser beams that can be utilized as the scale for measurement of an angular displacement. By detecting the change in peak frequency in optical spectra obtained by the photodetector, a small angular displacement can be measured. In order to verify the feasibility of proposed method, an experimental setup has been constructed. At first, an experiment has been carried out to investigate the effect of chromatic dispersion by the collimator objective employed in the developed setup. The experimental result has demonstrated that the change in focal length of approximetely $15 \mu \mathrm{m}$ can be generated by using the mode-locked femtosecond laser whose optical spectrum is ranging from $185 \mathrm{THz}$ to $200 \mathrm{THz}$. Results of the extended experiments have also demonstrated that the resolution of 0.23 arc-second can be expected by the developed optical setup.

It should be noted that this paper has focused on the proposal and verification of the measurement method as the first step of research. Further detained investigation on the determination of $\theta_{0}$, Quantitative evaluation of the fundamental characteristics of proposed system and optimization of the optical design of the proposed angle sensor, experiments for verifying the feasibility of proposed method for measurement of a larger angular displacement as well as the intensive analysis on the measurement uncertainty of the proposed method, will be carried out in future work.

\section{Acknowledgement}

This work was supported by the Japan Society for the Promotion of Science (JSPS).

\section{References}

Bryan, J. B., The Abbe Principle Revisited: An Updated Interpretation, Precision Engineering, Vol.1, No.3 (1979), pp.129-132.

Chen, Y. L., Shimizu, Y., Tamada, J., Kudo, Y., Madokoro, S., Nakamura, K. and Gao, W., Optical frequency domain angle measurement in a femtosecond laser autocollimator, Optics Express, Vol.25, No.14 (2017), pp.16725-16738.

Chong, A., Renninger, W. H. and Wise, F. W., Properties of normal-dispersion femtosecond fiber laser, Journal of the Optical Society of America B, Vol.25, No.2 (2008), pp.140-148.

Claus, D., Watson, J. and Rodenburg, J., Analysis and interpretation of the Seidel aberration coefficients in digital holography, Applied Optics, Vol.50, No.34 (2011), pp.H220-H229.

Ennos, A. E. and Virdee, M. S., High accuracy profile measurement of quasi-conical mirror surfaces by laser autocollimation, Journal of the International Societies for Precision Engineering and Nanotechnology, Vol. 4, No.1 (1982), pp. 5-8.

Gao, W., Precision Nanometrology Sensors and Measuring Systems for Nanomanufacturing, Springer, (2010).

Gao, W., Kim, S. W., Bosse, H., Haitjema, H., Chen, Y. L., Lu, X. D., Knapp, W., Weckenmann, A., Estler, W. T. and Kunzmann, H., Measurement technologies for precision positioning, CIRP Annals-Manufacturing Technology, Vol.64, No.2 (2015), pp.773-796.

Gao, W., Saito, Y., Muto, H., Arai, Y. and Shimizu, Y., A three-axis autocollimator for detection of angular error motions of a precision stage, CIRP Annals-Manufacturing Technology, Vol.60, No1. (2011), pp.515-518.

Hecht, E., Optics $5^{\text {th }}$ Ed. (2017) Pearson.

Holzwarth, R., Udem, Th., Hansch, T. W., Knight, J. C., Wadsworth, W. J. and Russell, P. St. J., Optical Frequency Synthesizer for Precision Spectroscopy, Physical Review Letters, Vol.85, No.11 (2000), pp.2264-2267. 
Ishikawa, K., Takamura, T., Xiao, M., Takahashi, S. and Takamasu, K., Profile measurement of aspheric surfaces using scanning deflectometry and rotating autocollimator with wide measuring range, Measurement Science and Technology, Vol.25, No.6 (2014), 064008.

Jones, D. J., Diddams, S. A., Ranka, J. K., Stentz, A., Windeler, R. S., Hall, J. L. and Cundiff, S. T., Carrier-Envelope Phase Control of Femtosecond Mode-Locked Lasers and Direct Optical Frequency Synthesis, Science, Vol.288, No.5466 (2000), pp.635-639.

Kim, S.W., Metrology: Combs Rule. Nature Photonics, Vol.3, No.6 (2009), pp. 313-314.

Minoshima, K. and Matsumoto, H., High-accuracy measurement of 240-m distance in an optical tunnel by use of a compact femtosecond laser, Vol.39, No.30 (2000), pp.5512-5517.

Moreaux, L., Sandre, O. and Mertz, J., Membrane imaging by second-harmonic generation microscopy, Journal of the Optical Society of America B, Vol.17, No.10 (2000), pp.1685-1694.

Okubo, S., Hsieh, Y. D., Inaba, H., Onae, A., Hashimoto, M. andYasui, T., Near-infrared broadband dual-frequency-comb spectroscopy with a resolution beyond the Fourier limit determined by the observation time window, Optics Express, Vol.23, No.26 (2015), pp.33184-33193.

Saito, S., Nilsonn, O. and Yamamoto, Y., Oscillation Center Frequency Tuning, Quantum FM Noise, and Direct Frequency Modulation Characteristics in External Grating Loaded Semiconductor Lasers, IEEE Journal of Quantum Electronics, Vol.18, No.6 (1982), pp.961-970.

Saito, Y., Gao, W., Kiyono, S., A Single Lens Micro-Angle Sensor, International Journal of Precision Engineering and Manufacturing, Vol.8, No.2 (2007), pp. 14-19.

Shimizu, Y., Tan, S. L., Murata, D., Maruyama, T., Ito, S., Chen, Y. L. and Gao, W., Ultra-sensitive angle sensor based on laser autocollimation for measurement of stage tilt motions, Optics Express, Vol.24, No.3 (2016), pp.2788-2805.

Tamada, J.,Kudo Y., Chen Y.L., Shimizu Y., and Gao W., Determination of the zero-position for an optical angle sensor, Journal of Advanced Mechanical Design, Systems, and Manufacturing, Vol.10, No.5 (2016), 16-00128 (10 pages).

Tan, B., Sivakumar, N. R. and Venkatakrishnan, K., Direct grating writing using femtosecond laser interference fringes formed at the focal point, Journal of Optics A: Pure and Applied Optics, Vol.7, No.4 (2005), pp.169-174.

Yasui, T., Minoshima, K. and Matsumoto, H., Three-dimensional shape measurement of a diffusing surface by use of a femtosecond amplifying optical Kerr gate, Applied Optics, Vol.39, No.1 (2000), pp.65-71. 